\title{
Cancer Type-Dependent Correlations between TP53 Mutations and Antitumor Immunity
}

\author{
Lin $\mathrm{Li}^{1,2,3}$, Mengyuan Li ${ }^{1,2,3}$, Xiaosheng Wang 1, 2, 3, * \\ ${ }^{1}$ Biomedical Informatics Research Lab, School of Basic Medicine and Clinical Pharmacy, China \\ Pharmaceutical University, Nanjing 211198, China \\ 2 Cancer Genomics Research Center, School of Basic Medicine and Clinical Pharmacy, China \\ Pharmaceutical University, Nanjing 211198, China \\ ${ }^{3}$ Big Data Research Institute, China Pharmaceutical University, Nanjing 211198, China \\ * Correspondence to: Xiaosheng Wang (E-mail address: xiaosheng.wang@cpu.edu.cn)
}

\section{Abstract}

Many studies have shown that TP53 mutations play a negative role in antitumor immunity. However, a few studies reported that TP53 mutations could promote antitumor immunity. To explain these contradictory findings, we analyzed five cancer cohorts from The Cancer Genome Atlas (TCGA) project. We found that TP53-mutated cancers had significantly higher levels of antitumor immune signatures than TP53-wildtype cancers in breast invasive carcinoma (BRCA) and lung adenocarcinoma (LUAD). In contrast, TP53-mutated cancers had significantly lower antitumor immune signature levels than TP53-wildtype cancers in stomach adenocarcinoma (STAD), colon adenocarcinoma (COAD), and head and neck squamous cell carcinoma (HNSC). Moreover, TP53-mutated cancers likely had higher tumor mutation burden (TMB) and tumor aneuploidy level (TAL) than TP53-wildtype cancers. However, the TMB differences were more marked between TP53-mutated and TP53-wildtype cancers than the TAL differences in BRCA and LUAD, and the TAL differences were more significant in STAD and COAD. Furthermore, we showed that TMB and TAL had a positive and a negative correlation with antitumor immunity and that TMB affected antitumor immunity more greatly than TAL did in BRCA and LUAD while TAL affected antitumor immunity more strongly than TMB in STAD and HNSC. These findings indicate that the distinct correlations between TP53 mutations and antitumor immunity in different cancer types are a consequence of the joint effect of the altered TMB and TAL caused by TP53 mutations on tumor immunity. Our data suggest that the TP53 mutation status could be a useful biomarker for cancer immunotherapy response depending on cancer types. 


\section{Introduction}

p53 is one of the most important tumor suppressor which is involved in regulation of a wide range of cancer-associated pathways, such as cell cycle, apoptosis, DNA damage repair, metabolism, immune and inflammation, angiogenesis, and metastasis (1). Its encoding gene TP53 is the most frequently-mutated gene in human cancers and the TP53 mutation is often associated with a worse prognosis in cancer (2). A number of studies have suggested that p53 plays an important role in tumor immune regulation (3-11). Notably, most of these prior studies consistently showed that p53 played a positive role in antitumor immunosurveillance $(3-8,10$, 11). As a result, $T P 53$ mutations often inhibited antitumor immunity and cancer immunotherapy response $(7,10)$. However, a few studies have reported that TP53 mutations could promote antitumor immune activity and immunotherapy response $(9,11,12)$. These contradictory findings suggest that the correlation between TP53 mutations and tumor immunity could be cancer type dependent.

p53 plays a key role in maintaining chromosomal/genomic stability (1). Accordingly, TP53 mutations may result in chromosomal/genomic instability that contributes to increased tumor mutation burden (TMB) and tumor aneuploidy level (TAL) (1). It has been shown that TMB tends to have a positive correlation with tumor immunity and immunotherapy response $(13,14)$ and TAL has a negative correlation with them (15). Thus, the alterations of tumor immunity and immunotherapy response could be a consequence of the joint effect of the altered TMB and TAL in TP53-mutated cancers relative to TP53-wildtype cancers. To prove this hypothesis, we analyzed five cancer cohorts from The Cancer Genome Atlas (TCGA) project (https://portal.gdc.cancer.gov/). The five cancer cohorts included breast invasive carcinoma 
(BRCA), lung adenocarcinoma (LUAD), stomach adenocarcinoma (STAD), colon adenocarcinoma (COAD), and head and neck squamous cell carcinoma (HNSC). We compared the enrichment levels of diverse immune signatures, TMB, and TAL between TP53-mutated and TP53-wildtype cancers and analyzed the correlations of TMB and TAL with immune signatures in each of the five cancer cohorts. Furthermore, we compared the immunotherapy response rates between TP53-mutated and TP53-wildtype cancers in a LUAD cohort (Dong cohort (9)) receiving anti-PD-1/PD-L1 immunotherapy. Moreover, we compared overall survival (OS) prognosis between TP53-mutated and TP53-wildtype cancers in the immunotherapy setting (Samstein cohort (13)) and in the non-immunotherapy setting (TCGA cohorts). This study revealed the mechanism underlying the distinct correlations of TP53 mutations with tumor immunity among different caner types.

\section{Materials and Methods}

\section{Materials}

The multi-omics (gene expression and gene somatic mutations) and clinical datasets for six TCGA cancer cohorts (BRCA, LUAD, STAD, COAD, HNSC, and lung squamous-cell carcinoma (LUSC)) were downloaded from the genomic data commons data portal (https://portal.gdc.cancer.gov/). The datasets for two cancer cohorts (Dong cohort (9) and Samstein cohort (13)) with anti-PD-1/PD-L1/CTLA-4 immunotherapy response-associated clinical data were obtained from their associated publications.

\section{Comparisons of the enrichment levels of immune signatures between TP53- mutated and TP53-wildtype cancers}


We quantified the enrichment level of an immune signature in a tumor sample by the singlesample gene-set enrichment analysis (ssGSEA) score (16) of the immune signature marker gene set. We analyzed three immune signatures, including CD8+ T cells (marker gene CD8A), NK cells (marker genes KLRC1 and KLRF1), and immune cytolytic activity (marker genes GZMA and PRF1). We compared the immune signature enrichment (ISE) levels between TP53-mutated and TP53-wildtype cancers using Mann-Whitney U test. In addition, we compared the ratios of immune-stimulatory signatures/immune-inhibitory signatures (CD8+/CD4+ regulatory T cells, pro-/anti-inflammatory cytokines, and M1/M2 macrophages) between TP53-mutated and TP53wildtype cancers using Mann-Whitney $U$ test. The ratios were the mean expression levels of immune-stimulatory signature marker genes divided by the mean expression levels of immuneinhibitory signature marker genes. Supplementary Table S1 presents the marker genes of these immune signatures.

\section{Comparisons of TMB and TAL between TP53-mutated and TP53-wildtype cancers}

For each tumor sample, we defined its TMB as the total number of somatic mutations in the tumor and TAL as the ploidy score which was assessed by the ABSOLUTE algorithm (17). We compared TMB and TAL between TP53-mutated and TP53-wildtype cancers using MannWhitney U test.

\section{Prediction of immune signature enrichment levels using TMB and TAL}

We used logistic regression to evaluate the contributions of TMB and TAL in predicting CD8+ T cell enrichment levels in cancer. The predictor TMB was log10 transformed and z-score 
standardized and TAL was z-score standardized. The CD8+ T cell enrichment level (high (upper third) versus low (bottom third)) was predicted.

\section{Comparisons of the enrichment levels of immune signatures between high- TMB and low-TMB cancers and between high-TAL and low-TAL cancers}

We compared the ISE levels between high-TMB ( $>$ median TMB) and low-TMB ( $<$ median TMB) cancers and between high-TAL (> median TAL) and low-TAL (< median TAL) cancers using Mann-Whitney U test.

\section{Survival analyses}

We compared OS prognosis between TP53-mutated and TP53-wildtype cancers in six TCGA cancer cohorts and two immunotherapy-associated cancer cohorts. We used Kaplan-Meier survival curves to show the OS time differences and the log-rank test to assess the significance of OS time differences.

\section{Results}

\section{Correlation between TP53 mutations and tumor immunity}

Interestingly, we found that the immune signatures (CD8+ T cells, NK cells, and immune cytolytic activity) exhibited significantly higher enrichment levels in TP53-mutated cancers than in TP53-wildtype cancers in BRCA and LUAD (Mann-Whitney $U$ test, $P<0.01$ ) (Figure 1A). However, almost all these immune signatures showed significantly lower enrichment levels in TP53-mutated cancers than in TP53-wildtype cancers in STAD, COAD, and HNSC (MannWhitney $\mathrm{U}$ test, $P<0.05$ ) (Figure $1 \mathrm{~A}$ ). Moreover, TP53-mutated cancers tended to have higher ratios of immune-stimulatory signatures/immune-inhibitory signatures (CD8+/CD4+ regulatory T cells, pro-/anti-inflammatory cytokines, and M1/M2 macrophages) than TP53-wildtype 
cancers in BRCA and LUAD (Mann-Whitney U test, $P<0.05$ ) (Figure 1B). In contrast, these ratios were likely lower in TP53-mutated cancers than in TP53-wildtype cancers in STAD, COAD, and HNSC (Figure 1B). Altogether, these results indicate that the correlation between TP53 mutations and tumor immunity varies depending on cancer types.

Furthermore, we found that numerous human leukocyte antigen (HLA) genes were significantly upregulated in TP53-mutated cancers relative to TP53-wildtype cancers in BRCA and LUAD (Student's $t$ test, $P<0.05$ ) (Figure $1 C$ ). In contrast, many HLA genes were significantly downregulated in TP53-mutated cancers versus TP53-wildtype cancers in STAD, COAD, and HNSC (Figure 1C). These results again indicate that the correlation between TP53 mutations and tumor immunity is cancer type dependent.

\section{Correlations of TP53 mutations with TMB and TAL}

We compared TMB and TAL between TP53-mutated and TP53-wildtype cancers in the TCGA cancer cohorts. As expected, both TMB and TAL were significantly higher in TP53-mutated cancers than in TP53-wildtype cancers except that TMB was lower in TP53-mutated COAD compared to TP53-wildtype COAD (Mann-Whitney U test, $P<0.05$ ) (Figure 2). The elevated TMB and TAL could be attributed to the increased chromosomal/genomic instability resulting from p53 inactivation in TP53-mutated cancers.

Interestingly, we found that the TMB differences were much more marked between TP53mutated and TP53-wildtype cancers compared to the TAL differences in BRCA and LUAD while the TAL differences were much more significant in STAD and COAD (Figure 2). It indicates that the altered degree of TMB and TAL resulting from TP53 mutations depends on 
cellular context. This finding supports the notion that p53 action and regulation are contextdependent (1).

\section{Correlations of TMB and TAL with tumor immunity}

We compared the enrichment levels of immune signatures between high-TMB and low-TMB cancers in each of the five TCGA cancer cohorts and found that these immune signatures were more highly enriched in high-TMB cancers than in low-TMB cancers (Mann-Whitney U test, $P<0.05$ ) (Figure 3A). The ratios of immune-stimulatory signatures/immune-inhibitory signatures were higher in high-TMB cancers than in low-TMB cancers (Mann-Whitney U test, $P<0.05$ ) (Figure 3A). Moreover, we compared the enrichment levels of immune signatures between highTAL and low-TAL cancers in each of the five TCGA cancer cohorts. We found that the immune signatures were more lowly enriched in high-TAL cancers than in low-TAL cancers (MannWhitney $U$ test, $P<0.05$ ) (Figure $3 \mathrm{~A}$ ). The ratios of immunostimulatory signature scores versus immunoinhibitory signature scores were lower in high-TAL cancers than in low-TAL cancers (Mann-Whitney U test, $P<0.05$ ) (Figure 3A). A number of HLA genes exhibited significantly higher expression levels in high-TMB cancers than in low-TMB cancers while significantly lower expression levels in high-TAL cancers than in low-TAL cancers (Student's $t$ test, $P<0.05$ ) (Supplementary Figure S1). These results were consistent with previous observation that tumor immunity had a positive correlation with TMB and an inverse correlation with TAL (15).

Furthermore, we used the logistic regression model with two predictors (TMB and TAL) to predict CD8+ T cell enrichment levels in each of the five TCGA cancer cohorts. We found that TMB was a stronger predictor than TAL in predicting CD8+ T cell enrichment levels in BRCA (TMB: $\beta=0.159, P=0.050$ versus TAL: $\beta=-0.072, P=0.422$ ) as well as in LUAD (TMB: $\beta=0.393$, 
$P=0.002$ versus TAL: $\beta=-0.389, P=0.002$ ) (Figure 3B). In contrast, TAL was a stronger predictor than TMB in predicting CD8+ T cell enrichment levels in STAD (TMB: $\beta=0.050, P=0.742$ versus TAL: $\beta=-0.418, P=0.007$ ) and in HNSC (TMB: $\beta=-0.041, P=0.732$ versus TAL: $\beta=-0.604$, $P=2.25 \times 10^{-6}$ ) (Figure 3C). These results indicate that TMB affects ISE to a greater degree than TAL does in BRCA and LUAD while TAL affects ISE more highly than TMB in STAD and HNSC. Since TMB and TAL are positively and negatively associated with tumor immunity, respectively, and TP53 mutations enhance both TMB and TAL, TP53 mutations may result in increased or reduced tumor immunity depending on which of TMB and TAL has a stronger impact on tumor immunity. It could explain why TP53-mutated cancers have higher tumor immunity than TP53-wildtype cancers in BRCA and LUAD while TP53-mutated cancers have lower tumor immunity than TP53-wildtype cancers in STAD and HNSC.

\section{Correlations of TP53 mutations with survival prognosis in cancer}

TP53 mutations have been associated with a worse survival in various cancers (2). However, TP53 mutations showed no a significant correlation with OS in BRCA (log-rank test, $P=0.162$ ). A possible explanation is that TP53 mutations promote antitumor immune response in BRCA (Figure 1A). In addition, we found that TP53 mutations were significantly associated with a worse OS in LUAD and HNSC (log-rank test, $P<0.05$ ) but showed no a significant correlation with OS in STAD, COAD, and gastrointestinal (GI) cancers. Furthermore, we examined the association between TP53 mutations and OS in these cancer types in a pan-cancer cohort (Samstein cohort (13)) receiving anti-PD-1/PD-L1/CTLA-4 immunotherapy. We found that TP53 mutations were significantly associated with a worse OS in COAD and GI cancers (logrank test, $P<0.05$ ) and exhibited no a significant correlation with OS in BRCA, LUAD, STAD, and HNSC in Samstein cohort. The negative correlation between TP53 mutations and OS in 
COAD and GI cancers in the immunotherapy setting rather than in the non-immunotherapy setting (Figure 4A) could be attributed to the better immunotherapy response in the TP53wildtype subtype than in the TP53-mutated subtype of COAD and GI cancers. On the other hand, the negative correlation between TP53 mutations and OS in LUAD in the non-immunotherapy setting rather than in the immunotherapy setting (Figure 4A) could be a consequence of the better immunotherapy response in TP53-mutated LUAD than in TP53-wildtype LUAD. This speculation was confirmed in a LUAD cohort (Dong cohort (9)) receiving anti-PD-1/PD-L1 immunotherapy where TP53-mutated LUAD had a 1.5 times higher immunotherapy response rate (57.1\%) than TP53-wildtype LUAD (37.5\%) and TP53-mutated LUAD showed a better OS than TP53-widtype LUAD (9). These results suggest that the association between TP53 mutations and immunotherapy response is cancer type dependent. This conclusion can be evidenced by two recent studies showing that TP53 mutations could promote immunotherapy response in LUAD (9) and inhibit immunotherapy response in melanoma (10). Furthermore, we found that $P D-L 1$ expression levels exhibited a significant positive correlation with TP53 mutations in BRCA and LUAD while a significant inverse correlation with TP53 mutations in STAD, COAD, and HNSC (Student's $t$ test, $P<0.05$ ) (Figure 4B). This could explain why TP53mutated LUAD had a better immunotherapy response than TP53-wildtype LUAD and TP53mutated COAD had a worse immunotherapy response than TP53-wildtype COAD since PD-L1 expression is a biomarker for anti-PD-1/PD-L1 immunotherapy response in cancer (18).

\section{Discussion}

A number of studies have revealed that cellular context is essential to p53 function and activity $(1,19)$. In this study, we demonstrated that regulation of tumor immunity by p53 was cancer type 
dependent. We found that the correlation between TP53 mutations and tumor immunity was positive in BRCA and LUAD while was negative in STAD, COAD, and HNSC. To our knowledge, this is the first study showing distinct correlations of TP53 mutations with tumor immunity in different cancer types. Interestingly, we found that even in the cancers originating from the same organ, the correlations of TP53 mutations with tumor immunity could be distinct. For example, the correlation between TP53 mutations and tumor immunity tended to be negative in LUSC (Supplementary Figure S2A) versus the positive correlation between TP53 mutations and tumor immunity in LUAD (Figure 1A). Moreover, TP53 mutations were associated with a worse OS in LUSC in the immunotherapy setting (Samstein cohort) (log-rank test, $P=0.046$ ) (Supplementary Figure S2B) while showed no a significant association with OS in LUAD in this cohort (log-rank test, $P=0.335$ ) (Figure 4A).

p53 is a significant protector of genome stability and TP53 mutations often comprise the p53 function in maintaining genome stability (20). As a result, TP53-mutated cancers tend to exhibit higher TMB and TAL than TP53-wildtype cancers (Figure 2) since genome instability leads to increased TMB and TAL in cancer. An exception was COAD in which TP53-mutated cancers had lower TMB and higher TAL than TP53-wildtype cancers (Figure 2). If the genome instability caused by TP53 mutations is restricted to local and small DNA sequences, such as microsatellite instability/deficient mismatch repair (MSI/dMMR), a higher degree of TMB differences would be presented between TP53-mutated and TP53-wildtype cancers. In contrast, if the genome instability caused by TP53 mutations is the alterations of chromosomal or large DNA sequences, such as DNA ploidy or copy number alterations, a higher degree of TAL differences would be shown between TP53-mutated and TP53-wildtype cancers. Because TMB 
and TAL have a positive and a negative correlation with tumor immunity, respectively, TP53 mutations may increase or reduce tumor immunity depending on the counteractive effect of TMB and TAL on tumor immunity (Figure 5). We confirmed this hypothesis by analyzing five TCGA cancer cohorts. We further demonstrated that the TP53 mutation status could be a positive or a negative predictor for cancer immunotherapy response depending on cancer types.

\section{Conclusions}

The correlations between TP53 mutations and tumor immunity and immunotherapy response are cancer type dependent. These findings have potential clinical implications for cancer immunotherapy.

\section{Disclosure of Potential Conflicts of Interest}

The authors declare that they have no competing interests.

\section{Authors' Contributions}

Conception and design: $X$. Wang

Development of methodology: X. Wang, L. Li, M. Li

Acquisition of data (provided animals, acquired and managed patients, provided facilities, etc.): L. Li, M. Li

Analysis and interpretation of data (e.g., statistical analysis, biostatistics, computational analysis): L. Li, M. Li, X. Wang

Writing, review, and/or revision of the manuscript: $X$. Wang

Administrative, technical, or material support (i.e., reporting or organizing data, constructing databases): L. $\mathrm{Li}, \mathrm{M} . \mathrm{Li}$

Study supervision: $X$. Wang

\section{Acknowledgments}

We thank Mr. Zehang Jiang from China Pharmaceutical University for help in data analyses. 


\section{References}

1. Kastenhuber ER \& Lowe SW (2017) Putting p53 in Context. Cell 170(6):1062-1078.

2. Wang X \& Sun Q (2017) TP53 mutations, expression and interaction networks in human cancers. Oncotarget 8(1):624-643.

3. Zitvogel L \& Kroemer G (2015) CANCER. A p53-regulated immune checkpoint relevant to cancer. Science 349(6247):476-477.

4. Textor S, et al. (2011) Human NK cells are alerted to induction of p53 in cancer cells by upregulation of the NKG2D ligands ULBP1 and ULBP2. Cancer research 71(18):59986009.

5. Shatz M, Menendez D, \& Resnick MA (2012) The human TLR innate immune gene family is differentially influenced by DNA stress and p53 status in cancer cells. Cancer research 72(16):3948-3957.

6. Guo G, Yu M, Xiao W, Celis E, \& Cui Y (2017) Local activation of p53 in the tumor microenvironment overcomes immune suppression and enhances antitumor immunity. Cancer research.

7. Jiang Z, Liu Z, Li M, Chen C, \& Wang X (2018) Immunogenomics Analysis Reveals that TP53 Mutations Inhibit Tumor Immunity in Gastric Cancer. Translational oncology 11(5):1171-1187.

8. Wang B, Niu D, Lai L, \& Ren EC (2013) p53 increases MHC class I expression by upregulating the endoplasmic reticulum aminopeptidase ERAP1. Nature communications 4:2359.

9. Dong ZY, et al. (2017) Potential Predictive Value of TP53 and KRAS Mutation Status for Response to PD-1 Blockade Immunotherapy in Lung Adenocarcinoma. Clinical cancer research : an official journal of the American Association for Cancer Research 23(12):3012-3024.

10. Xiao W, et al. (2018) TP53 Mutation as Potential Negative Predictor for Response of Anti-CTLA-4 Therapy in Metastatic Melanoma. EBioMedicine 32:119-124.

11. Ham SW, et al. (2019) TP53 gain-of-function mutation promotes inflammation in glioblastoma. Cell Death Differ 26(3):409-425.

12. Liu Z, et al. (2019) TP53 Mutations Promote Immunogenic Activity in Breast Cancer. Journal of Oncology 2019(Article ID 5952836):1-19. 
13. Samstein RM, et al. (2019) Tumor mutational load predicts survival after immunotherapy across multiple cancer types. Nature genetics 51(2):202-206.

14. Goodman AM, et al. (2017) Tumor Mutational Burden as an Independent Predictor of Response to Immunotherapy in Diverse Cancers. Molecular cancer therapeutics 16(11):2598-2608.

15. Davoli T, Uno H, Wooten EC, \& Elledge SJ (2017) Tumor aneuploidy correlates with markers of immune evasion and with reduced response to immunotherapy. Science 355(6322).

16. Hanzelmann S, Castelo R, \& Guinney J (2013) GSVA: gene set variation analysis for microarray and RNA-seq data. BMC bioinformatics 14:7.

17. Carter SL, et al. (2012) Absolute quantification of somatic DNA alterations in human cancer. Nature biotechnology 30(5):413-421.

18. Patel SP \& Kurzrock R (2015) PD-L1 Expression as a Predictive Biomarker in Cancer Immunotherapy. Molecular cancer therapeutics 14(4):847-856.

19. Andrysik Z, et al. (2017) Identification of a core TP53 transcriptional program with highly distributed tumor suppressive activity. Genome research 27(10):1645-1657.

20. Eischen CM (2016) Genome Stability Requires p53. Cold Spring Harbor perspectives in medicine 6(6).

\section{Figure legends}

Figure 1. Correlation between TP53 mutations and tumor immunity. A. Three immune signatures (CD8+ T cells, NK cells, and immune cytolytic activity) show significantly higher enrichment levels (ssGSEA scores) in TP53-mutated cancers than in TP53-wildtype cancers in BRCA and LUAD and lower enrichment levels in TP53-mutated cancers than in TP53-wildtype cancers in STAD, COAD, and HNSC (Mann-Whitney $U$ test, $P<0.05$ ). B. The ratios of immune-stimulatory signatures/immune-inhibitory signatures (CD8+/CD4+ regulatory T cells, pro-/anti-inflammatory cytokines, and M1/M2 macrophages) tend to be higher in TP53-mutated cancers than in TP53-wildtype cancers in BRCA and LUAD and to be lower in TP53-mutated 
cancers than in TP53-wildtype cancers in STAD, COAD, and HNSC (Mann-Whitney U test, $P<0.05)$. C. Many HLA genes have higher expression levels in TP53-mutated cancers than in TP53-wildtype cancers in BRCA and LUAD and have lower expression levels in TP53-mutated cancers than in TP53-wildtype cancers in STAD, COAD, and HNSC (Student's $t$ test, $P<0.05$ ). ssGSEA: sample gene-set enrichment analysis (16). BRCA: breast invasive carcinoma. LUAD: lung adenocarcinoma. STAD: stomach adenocarcinoma. COAD: colon adenocarcinoma. HNSC: head and neck squamous cell carcinoma. HLA: human leukocyte antigen. ns: not significant. FC: fold change of mean gene expression levels (TP53-mutated cancers/TP53-wildtype cancers). * $P<0.05$, ** $P<0.01, * * * P<0.001$. They also apply to following figures.

Figure 2. Comparisons of TMB and TAL between TP53-mutated and TP53-wildtype cancers. Both TMB and TAL are significantly higher in TP53-mutated cancers than in TP53wildtype cancers except that TMB is lower in TP53-mutated COAD than in TP53-wildtype COAD (Mann-Whitney U test, $P<0.05$ ). The TMB differences are much more marked between TP53-mutated and TP53-wildtype cancers compared to the TAL differences in BRCA and LUAD while the TAL differences are much more significant in STAD and COAD (a smaller $P$ value indicates a more significant difference). TMB: tumor mutation burden. TAL: tumor aneuploidy level. They also apply to following figures.

Figure 3. Correlations of TMB and TAL with tumor immunity. A. The immune signature enrichment levels and the ratios of immune-stimulatory signatures/immune-inhibitory signatures are higher in high-TMB (TMB > median) cancers than in low-TMB (TMB < median) cancers and are lower in high-TAL (TAL > median) cancers than in low-TAL (TAL < median) cancers (Mann-Whitney U test, $P<0.05$ ). FC: fold change of mean immune signature enrichment levels or ratios. B. Logistic regression analysis shows that TMB is a stronger predictor than TAL in predicting CD8+ T cell enrichment levels in BRCA and LUAD and TAL a stronger predictor than TMB in predicting CD8+ T cell enrichment levels in STAD and HNSC.

Figure 4. Correlations of TP53 mutations with survival prognosis in cancer. A. KaplanMeier survival curves show that TP53 mutations have a negative correlation with overall survival (OS) in COAD and gastrointestinal cancers in the immunotherapy setting (Samstein cohort (13)) but have no a significant correlation with OS in the non-immunotherapy setting (TCGA cohorts) and that TP53 mutations have a negative correlation with OS in LUAD in the non-immunotherapy setting but have no a significant correlation with OS in the immunotherapy 
setting. Log-rank test $P$ values are shown. B. $P D-L 1$ expression levels are significantly higher in TP53-mutated cancers than in TP53-wildtype cancers in BRCA and LUAD and are significantly lower in TP53-mutated cancers than in TP53-wildtype cancers in STAD, COAD, and HNSC (Student's $t$ test, $P<0.05$ ).

Figure 5. The mechanism underlying the distinct correlations of TP53 mutations with tumor immunity among different caner types. MSI: microsatellite instability. dMMR: deficient mismatch repair. CNAs: copy number alterations.

\section{Supplementary data}

Supplementary Table S1. The gene sets that represent different immune signatures.

Supplementary Figure S1. A number of HLA genes show significantly higher expression levels in high-TMB cancers than in low-TMB cancers and significantly lower expression levels in high-TAL cancers than in low-TAL cancers (Student's $t$ test, $P<0.05$ ).

Supplementary Figure S2. Correlations of TP53 mutations with tumor immunity and overall survival (OS) prognosis in LUSC. A. The correlation between TP53 mutations and tumor immunity tends to be negative in LUSC. B. Kaplan-Meier survival curves show that TP53 mutations are associated with a worse OS in LUSC in the immunotherapy setting (Samstein cohort (13)) (log-rank test, $P=0.046$ ) and are associated with a better OS in LUSC in the nonimmunotherapy setting (TCGA cohort). LUSC: lung squamous-cell carcinoma. 

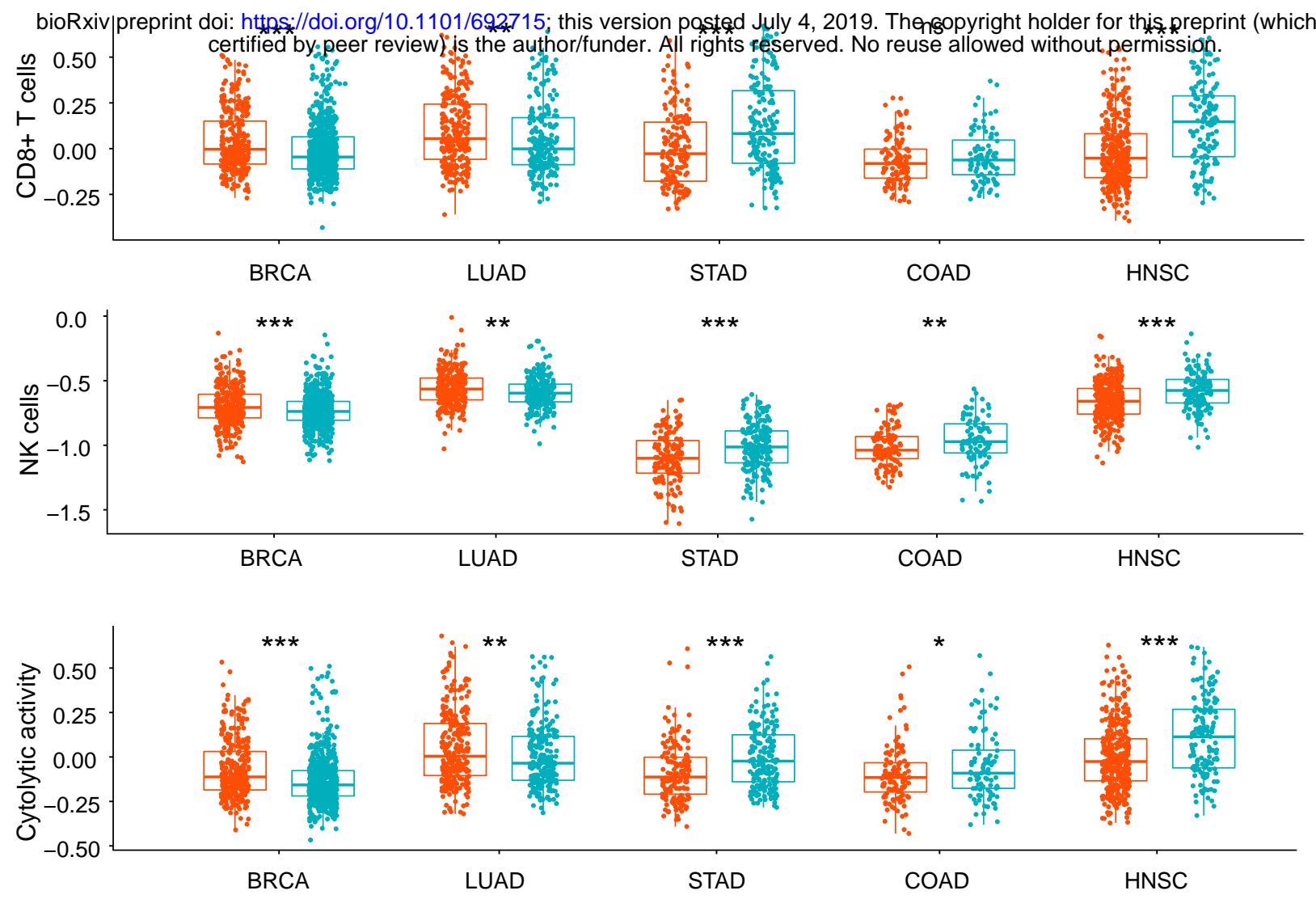

B

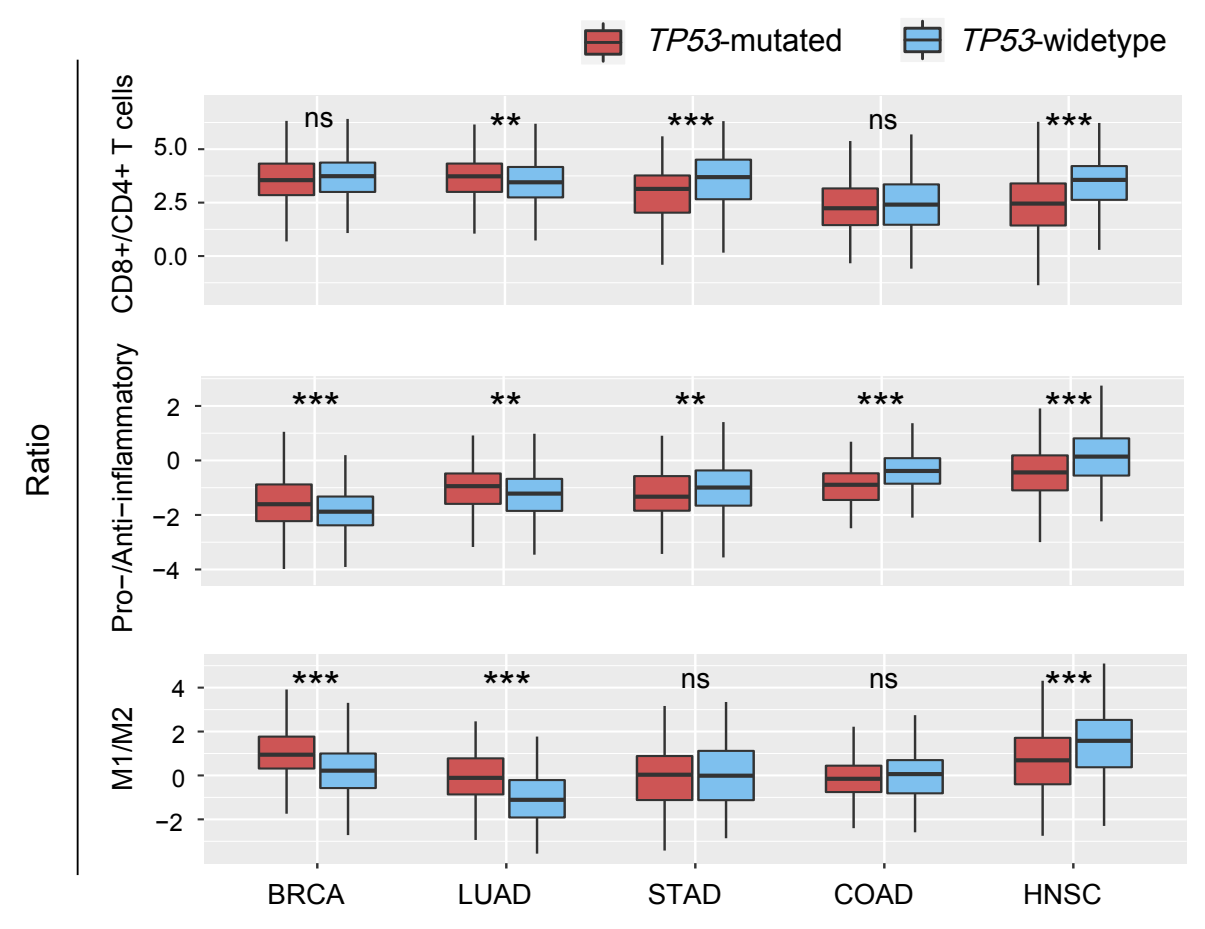

TP53-mutated vs. TP53-widetype (Student's $t$ test)

\begin{tabular}{|c|c|c|c|c|}
\hline$H L A-L$ & $* * *$ & ns & ns & \multirow{25}{*}{$\begin{array}{r}\log _{2} F C \\
\quad 1.0\end{array}$} \\
\hline$H L A-J$ & ns & ns & ns & \\
\hline$H L A-H$ & $* * *$ & ns & ns & \\
\hline$H L A-G$ & $* * *$ & ns & ns & \\
\hline$H L A-F$ & $* * *$ & ns & ns & \\
\hline$H L A-E$ & $* * *$ & * & ns & \\
\hline$H L A-D R B 6$ & ** & * & $* * *$ & \\
\hline$H L A-D R B 5$ & ns & $* *$ & $* * *$ & \\
\hline$H L A-D R B 1$ & $* *$ & $* *$ & $* * *$ & \\
\hline$H L A-D R A$ & $* *$ & $* * *$ & $* * *$ & \\
\hline$H L A-D Q B 2$ & ns & $* *$ & $* *$ & \\
\hline$H L A-D Q B 1$ & $* *$ & * & $* * *$ & \\
\hline$H L A-D Q A 2$ & $* *$ & $* *$ & $* * *$ & \\
\hline$H L A-D Q A 1$ & $* * *$ & $* * *$ & $* * *$ & \\
\hline$H L A-D P B 2$ & $* \star *$ & ** & $* * *$ & \\
\hline$H L A-D P B 1$ & $\mathrm{~ns}$ & $* * *$ & $* * *$ & \\
\hline$H L A-D P A 1$ & ns & $* * *$ & $* * *$ & \\
\hline$H L A-D O B$ & $* * *$ & ** & $* * *$ & \\
\hline$H L A-D O A$ & ns & $* * *$ & $* * *$ & \\
\hline$H L A-D M B$ & ** & $* * *$ & $* * *$ & \\
\hline$H L A-D M A$ & $* * *$ & $* * *$ & $\star * *$ & \\
\hline$H L A-C$ & $* * *$ & ns & ns & \\
\hline$H L A-B$ & $* * *$ & ns & ns & \\
\hline \multirow[t]{2}{*}{$H L A-A$} & $* * *$ & ns & ns & \\
\hline & BRCA & STAD & HNSC & \\
\hline
\end{tabular}


$P=1.86 \mathrm{e}-24$

$P=0.019$

$P=0.014$

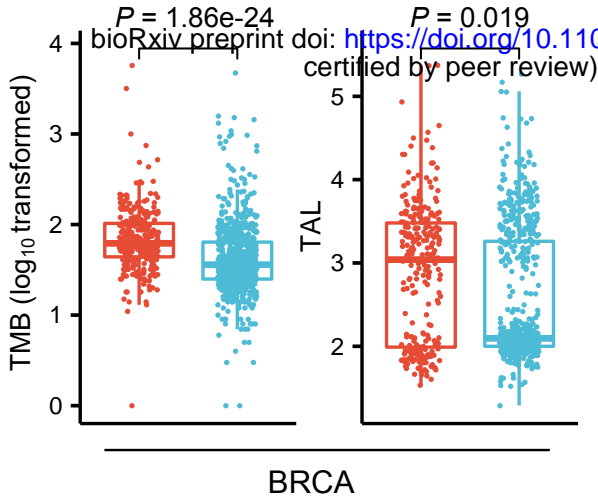

$P=2.80 \mathrm{e}-23$
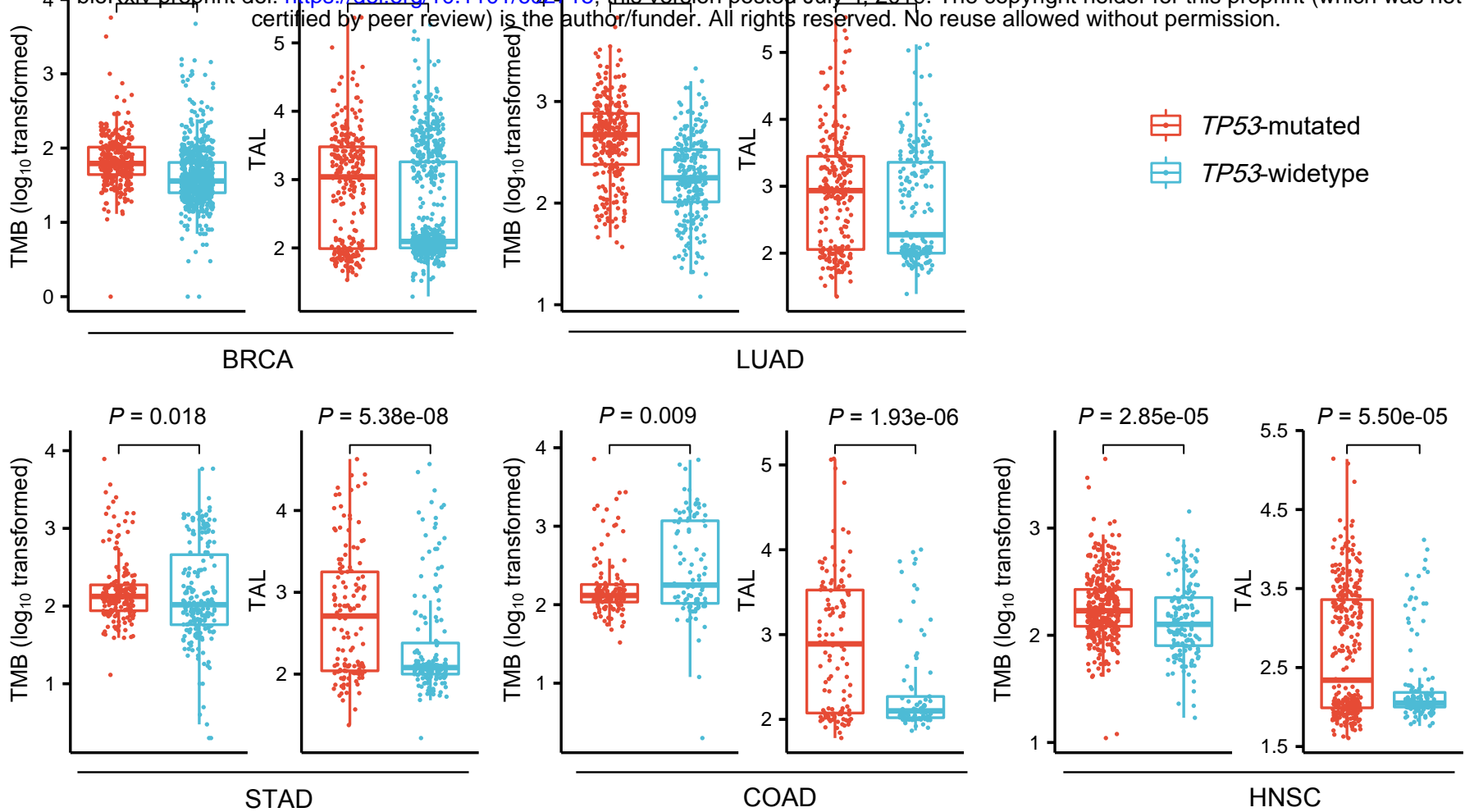
A

\section{high-TMB vs. low-TMB}

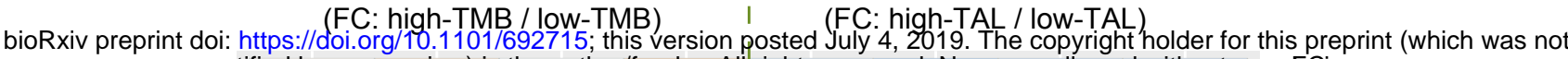
certified by peer review) is the author/funder. All rights reserved. No reuse allowed without permßsion.

\begin{tabular}{r|c|c|c|}
\hline CD8+ T cells & $*$ & $* * *$ & ns \\
\hline NK cells & $* *$ & $* *$ & ns \\
\hline & $* * *$ & $* *$ & $* *$ \\
\hline
\end{tabular}

CD8+/CD4+ regulatory $T$ cells

Pro-/Anti-inflammatory cytokines

M1/M2 macrophages

ns

$* * *$
ns
$* *$
$\searrow ্$
$\searrow$

ns
$* * *$
$* * *$
क्षे

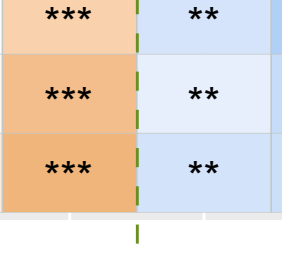
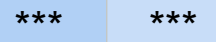

$* * *$

$\star * *$

$* * *$

$* * *$

$\star * *$

***

**

$* * *$
$\mathrm{~ns}$
$\mathrm{~ns}$
$\stackrel{े}{\omega}$

$0^{0} e_{2}^{2}$

B

\section{CD8+ T cell enrichment level (high vs. low) $\sim$ TMB + TAL}

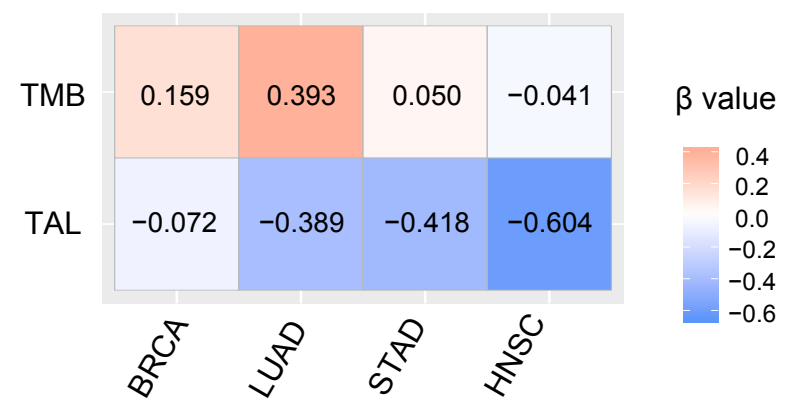


COAD

COAD

Gastrointestinal cancers

Gastrointestinal cancers

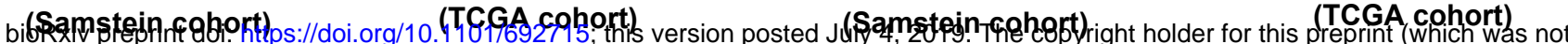

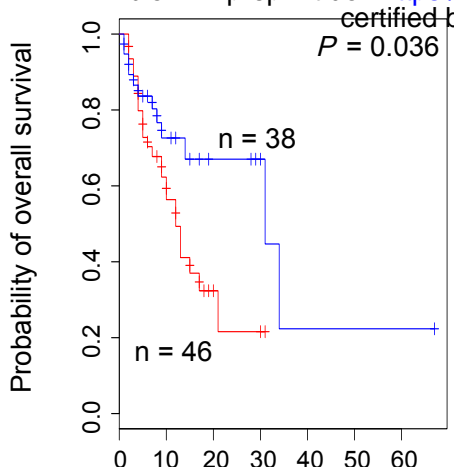

LUAD

(Samstein cohort)

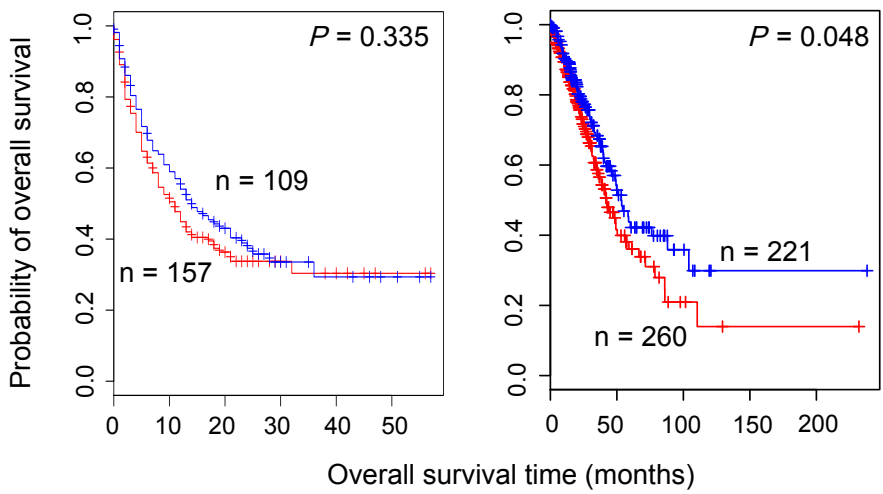

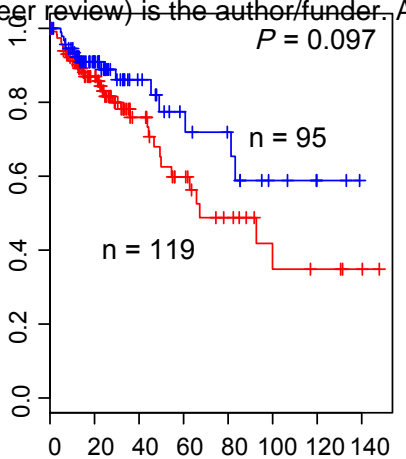
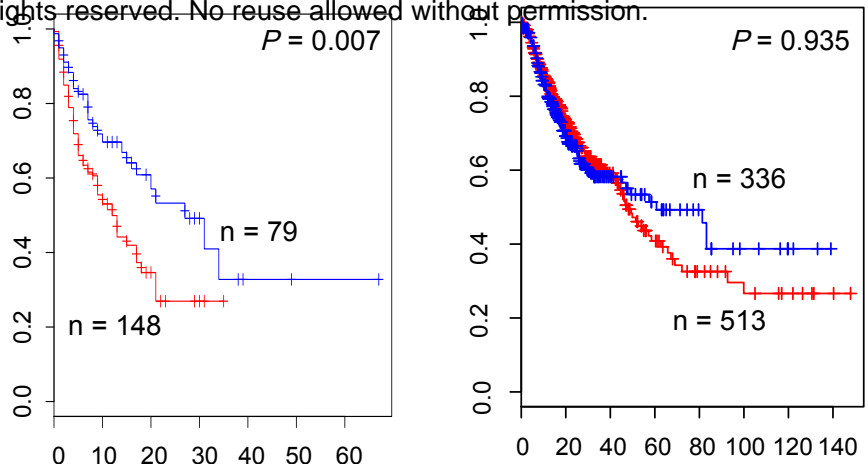

Overall survival time (months)
LUAD (TCGA cohort)

TP53-mutated

TP53-widetype
B
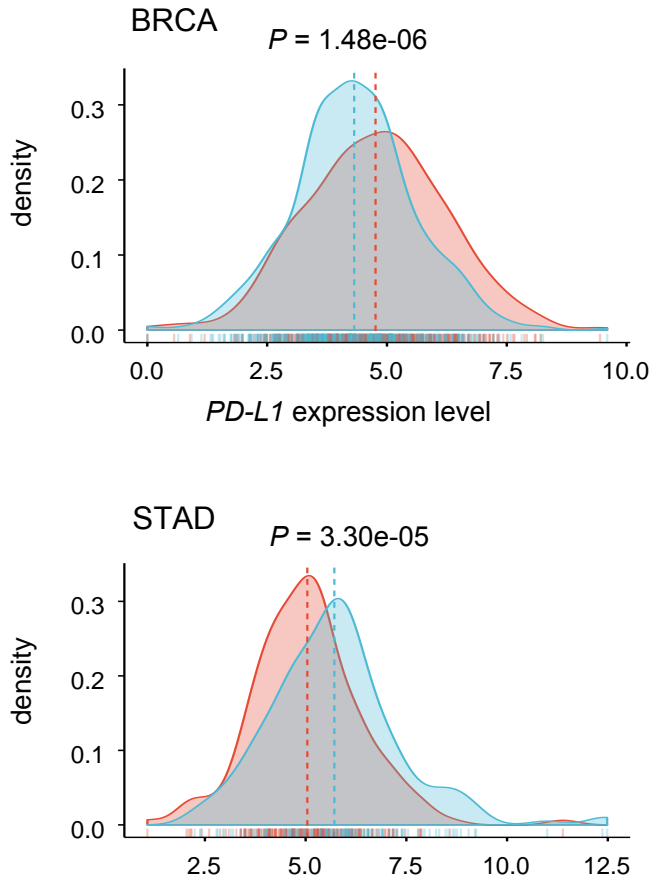

$P D$ - L1 expression level

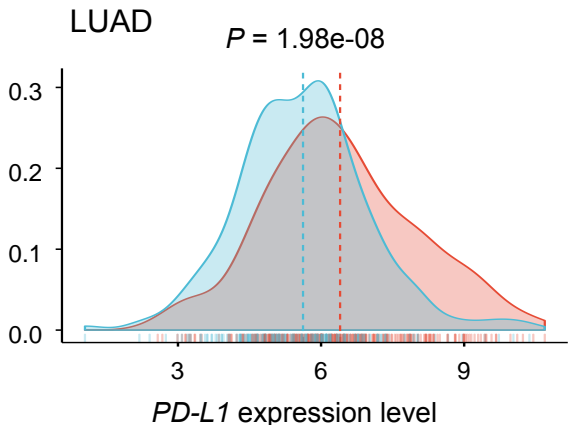

COAD

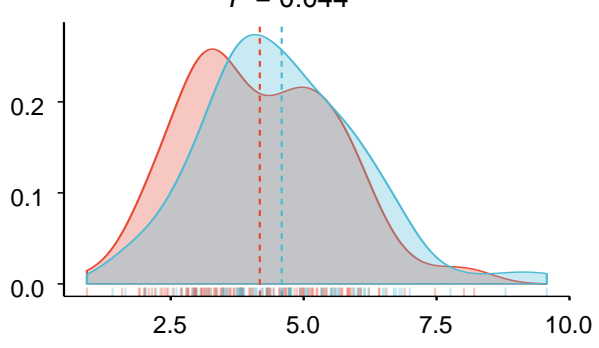

$P D-L 1$ expression level
TP53-mutated

TP53-widetype

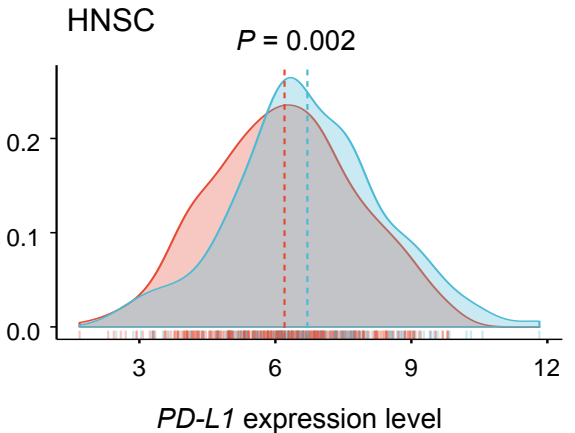




\section{TP53 mutations}

bioRxiv preprint doi: https://doi.org/10.1101/692715; this version posted July 4, 2019. The copyright holder for this preprint (which was not certified by peer review) is the author/funder. All righis reserved. No reuse allowed without permission.

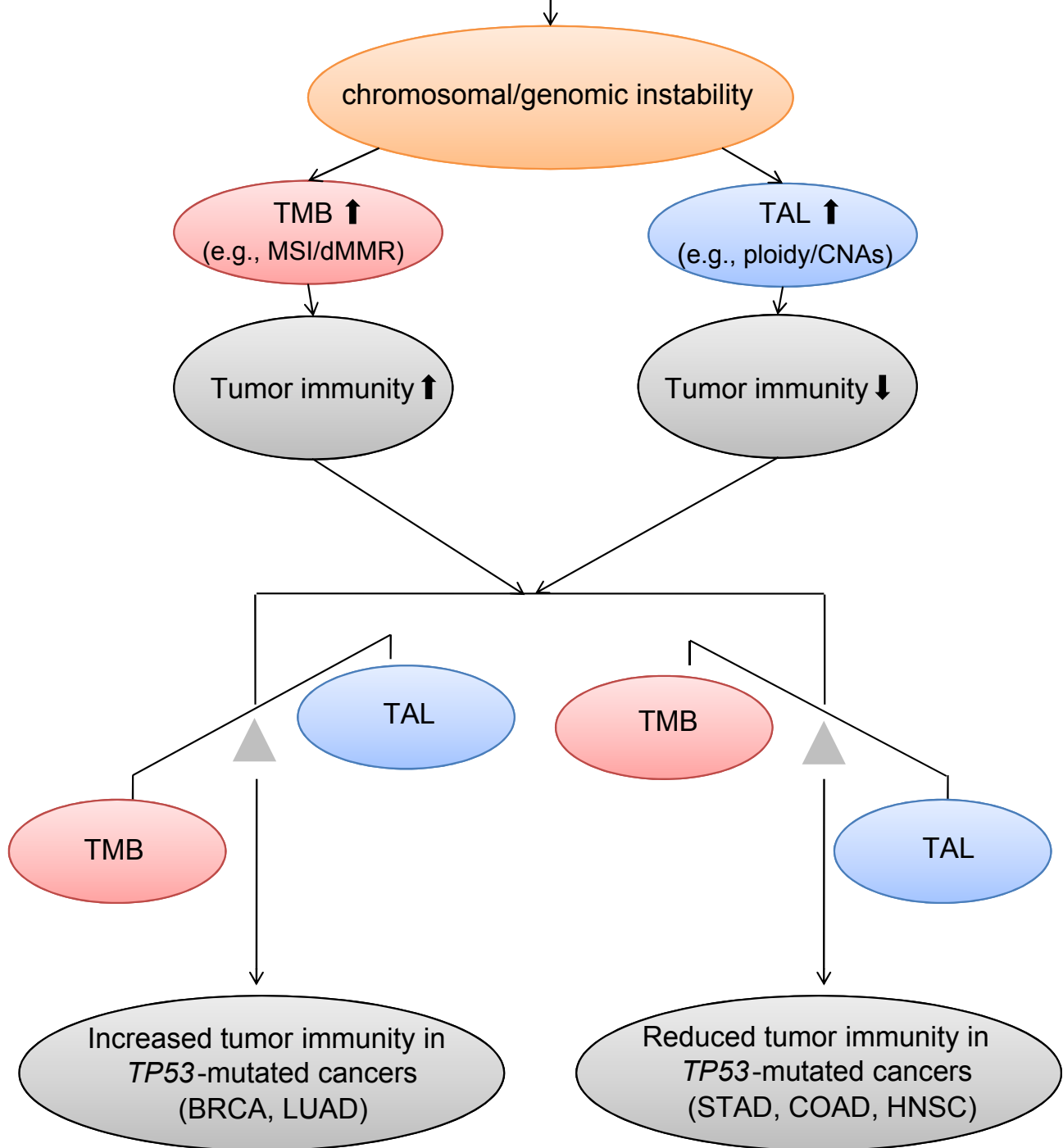

